\title{
Abnormal Low-Frequency Oscillations Reflect Trait-Like Pain Ratings in Chronic Pain Patients Revealed through a Machine Learning Approach
}

\author{
Anton Rogachov, ${ }^{1,2}$ Joshua C. Cheng, ${ }^{1,2}$ Kasey S. Hemington, ${ }^{1,2}$ Rachael L. Bosma, ${ }^{1}$ Junseok A. Kim, ${ }^{1,2}$ \\ Natalie R. Osborne, ${ }^{1,2}$ Robert D. Inman, ${ }^{2,3,4}$ and $\odot$ Karen D. Davis ${ }^{1,2,5}$ \\ ${ }^{1}$ Division of Brain, Imaging, and Behaviour-Systems Neuroscience, Krembil Brain Institute, Krembil Research Institute, Toronto Western Hospital, \\ University Health Network, Toronto, Ontario M5T 2S8, Canada, ${ }^{2}$ Institute of Medical Science, University of Toronto, Toronto, Ontario M5S 2J7, Canada, \\ ${ }^{3}$ Division of Genetics and Development, Krembil Research Institute, University Health Network, Toronto, Ontario M5T 2S8 Canada, and Departments of \\ ${ }^{4}$ Medicine and ${ }^{5}$ Surgery, University of Toronto, Toronto, Ontario M5T 1P5, Canada
}

Measures of moment-to-moment fluctuations in brain activity of an individual at rest have been shown to be a sensitive and reliable metric for studying pathological brain mechanisms across various chronic pain patient populations. However, the relationship between pathological brain activity and clinical symptoms are not well defined. Therefore, we used regional BOLD signal variability/amplitude of low-frequency oscillations (LFOs) to identify functional brain abnormalities in the dynamic pain connectome in chronic pain patients that are related to chronic pain characteristics (i.e., pain intensity). Moreover, we examined whether there were sex-specific attributes of these functional brain abnormalities and whether functional brain abnormalities in patients is related to pain intensity characteristics on different time scales. We acquired resting-state functional MRI and quantified frequency-specific regional LFOs in chronic pain patients with ankylosing spondylitis. We found that patients exhibit frequency-specific aberrations in LFOs. Specifically, lower-frequency (slow-5) abnormalities were restricted to the ascending pain pathway (thalamus and S1), whereas higher-frequency abnormalities also included the default mode (i.e., posterior cingulate cortex; slow-3, slow-4) and salience (i.e., mid-cingulate cortex) networks (slow-4). Using a machine learning approach, we found that these abnormalities, in particular within higher frequencies (slow-3), can be used to make generalizable inferences about patients' average pain ratings (trait-like pain) but not current (i.e., state-like) pain levels. Furthermore, we identified sex differences in LFOs in patients that were not present in healthy controls. These novel findings reveal mechanistic brain abnormalities underlying the longer-lasting symptoms (trait pain intensity) in chronic pain.

Key words: bold variability; chronic pain; fMRI; low-frequency oscillations

\section{Significance Statement}

Measures of moment-to-moment fluctuations in brain activity of an individual at rest have been shown to be a reliable metric for studying functional brain associated with chronic pain. The current results demonstrate that dysfunction in these intrinsic fluctuations/oscillations in the ascending pain pathway, default mode network, and salience network during resting state display sex differences and can be used to make inferences about trait-like pain intensity ratings in chronic pain patients. These results provide robust and generalizable implications for investigating brain mechanisms associated with longer-lasting/trait-like chronic pain symptoms.

\section{Introduction}

The temporal dimension of chronic pain is often overlooked, yet patients commonly report that their symptoms can fluctuate from moment to moment, and even on the order of hours and days. The mechanisms that contribute to chronic, fluctuating levels of pain and how they differ across individuals provide a 
window into underlying etiologies and potential treatment approaches. However, most brain imaging studies of chronic pain do not distinguish pain at the time of the study (state pain) from average pain experienced over time (trait pain). State and trait pain can reflect different aspects of chronic pain conditions.

One chronic pain condition that affects primarily younger adults devoid of comorbidities commonly seen in other chronic pain disorders is ankylosing spondylitis (AS). AS is a form of arthritis resulting from inflammation of the sacroiliac joint, resulting in debilitating lower back pain (Bidad et al., 2017). These patients have aching, tender and sore pain that can be shooting or stabbing (Wu et al., 2013), of intensity ebbs and flows throughout the day (Sieper et al., 2002).

One approach to investigate temporal mechanisms underlying chronic pain is to quantify low-frequency oscillations (LFOs) of brain activity during resting-state functional MRI (fMRI). This can be measured by BOLD signal variability, the SD of the BOLD signal time course (Garrett et al., 2010; Rogachov et al., 2016). We recently demonstrated that regional BOLD signal variability is a sensitive indicator of individual differences of pain sensitivity and coping in healthy individuals (Rogachov et al., 2016). Moreover, others have reported abnormalities in LFOs in chronic pain patients with back pain (Baliki et al., 2011), osteoarthritis (Baliki et al., 2014), migraine (Hodkinson et al., 2016), and neuropathic pain (Alshelh et al., 2016), but they reported conflicting findings. Baliki et al. (2011) reported that LFO abnormalities dynamically track fluctuations in patients' ongoing (state-like) pain during fMRI in low back pain patients; however, later work has also shown LFO abnormalities in low back pain and osteoarthritis to be related to more longer-lasting/trait-like pain characteristics (i.e., disease duration; Baliki et al., 2014). Likewise, recent work from our group has shown unique brain connectivity patterns associated with chronic pain characteristics of different time scales that represent trait-like and state-like pains (Cheng et al., 2018). In addition, an important consideration to LFO studies is frequency-specific and sex-related effects. Chronic pain-related disruption on LFOs and subsequent correlations to ongoing levels of pain (pain state; Baliki et al., 2011) and longer-lasting features (disease duration; Baliki et al., 2014) were found exclusively within higher frequencies $(0.12-0.20 \mathrm{~Hz}$; slow-3). Similarly, Hong et al. (2013) reported differences in frequency-specific alterations in resting-state brain oscillations between men and women patients with chronic visceral pain.

Therefore, the primary aims of this study were to (1) identify abnormalities in slow-3, -4 , and -5 BOLD signal variability/LFOs associated with chronic pain in patients with AS and (2) determine whether frequency-specific abnormalities are related to pain intensity ratings. We also considered whether these abnormalities related to patients' pain intensity levels on different time scales [current pain (state-like characteristic) vs average pain intensity over $7 \mathrm{~d}$ (trait-like characteristics)] and whether these abnormalities are impacted by sex. Toward these goals, we collected resting-state fMRI from chronic pain patients with AS ( $n=$ 71 ) and from 62 age- and sex-matched healthy controls. We also asked patients to rate their average pain over the previous week (trait-like pain) as well as the intensity of their pain immediately before the scan (current pain levels that reflects state-like pain). Importantly, we used a machine learning algorithm to determine whether functional brain differences between patients and controls can be used to make generalizable inferences related to patients' pain intensity ratings. We hypothesized that AS patients would show increased LFOs compared with healthy controls (HCs) and that higher-frequency (slow-3) LFOs would be related to their trait-like pain represented by average pain intensity over the previous week.

\section{Materials and Methods}

Subjects. The study consisted of 71 right-handed chronic pain patients diagnosed with AS (51 men, 20 women; average age \pm SD, $34.9 \pm 11.1$; $18-61$ years old) and 62 right-handed HCs ( 43 men, 19 women; average age $\pm \mathrm{SD}, 34.0 \pm 11 ; 18-55$ years old) who underwent a neuroimaging session and psychophysical testing. The AS cohort reported an average $( \pm S D)$ years living with pain of 15.5 (9.8), which ranged from 2 to 38 years. Although AS pain is classically associated with pathology localized to the sacroiliac joint (lower back), many patients reported bilateral pain radiating into their lower extremities, as well as other joints (e.g., knees and wrists). Forty-two of the patients were being treated with antiTNF- $\alpha$ (biologics) medication (i.e., Embrel, Humira, Remicade, and Simponi), and 27 were treated with nonsteroidal anti-inflammatory drugs (i.e., Voltaren, Naprosyn, and Celebrex). All participants provided informed written consent to procedures approved by the University Health Network research ethics board. Patients with chronic pain were recruited from the Spondylitis clinic at Toronto Western Hospital. The inclusion criteria for the chronic pain patients was a diagnosis of AS according to the modified New York criteria (van der Linden et al., 1984). Exclusion criteria for patients and HC participants were as follows: (1) a previous diagnosis of a psychiatric, neurological, or metabolic disorder; (2) major surgery in the past 2 years; (3) serious infection (associated with hospitalization or intravenous antibiotics) within 4 weeks of testing; and (4) any standard contraindications to MRI scanning (i.e., pregnancy, metal in the body, claustrophobia).

Neuroimaging acquisition. Brain imaging data were acquired with a 3T MRI scanner fitted with an eight-channel phase-array head coil (GE Medical Systems). For each study participant, we collected a highresolution T1-weighted anatomical scan (3D IR-FSPGR sequence; 180 axial slices; TR, $7.8 \mathrm{~ms}$; TE, $3 \mathrm{~ms}$; flip angle, $15^{\circ}$; $256 \times 256$ matrix; $1 \times$ $1 \times 1 \mathrm{~mm}$ voxels) and a $9 \mathrm{~min} .14 \mathrm{~s} \mathrm{~T} 2^{*}$-weighted resting-state fMRI scan (echo-planar imaging sequence; 36 slices; TR, $2000 \mathrm{~ms}$; TE, $30 \mathrm{~ms} ; 64 \times$ 64 matrix; $3.125 \times 3.125 \times 4 \mathrm{~mm}$ voxels). During the resting-state fMRI scan, the subjects were instructed to "close your eyes, do not try to think about anything in particular; do not fall asleep." Two subjects (both AS patients) were excluded from further analyses because of signal dropout within the orbitofrontal cortex, leaving a total of 69 subjects.

Questionnaires and pain ratings. All study subjects completed psychological/personality questionnaires, including the Beck Depression Inventory (BDI; Beck et al., 1961) and the State/Trait Anxiety Inventory (Spielberger et al., 1983), and patients filled out additional pain-specific assessments such as their average pain intensity over the past week and their current level of pain before imaging ( 0 , no pain; 10 , worse pain imaginable). Average pain intensity ratings were collected from 65 of 69 AS patients included in the study. Nine patients [all of whom were treated with anti-TNF- $\alpha$ (biologics) medication] reported an average weekly pain of 0 of 10 . As the goal of this study was to investigate the relationship between LFOs and pain intensity, these nine patients were removed, leaving a total of 56 AS patients in the main analysis. All 56 patients provided ratings of average pain; however, only 50 of 56 patients also provided a current pain intensity rating.

Preprocessing of $f M R I$ data. Data were preprocessed using the $\mathrm{AMRI}$ Expert Analysis Tool (FEAT) in FSL version 5.0 (Jenkinson et al., 2012). First, we deleted the first four volumes of the resting-state scan, and then we removed nonbrain voxels using the Brain Extraction Tool (BET) within FEAT and performed motion correction (MCFLIRT). T1-weighted anatomical images were skull stripped using optiBET (Lutkenhoff et al., 2014). The functional images were registered to the skull-stripped, T1-weighted anatomical images using FLIRT [rigid-body transformation with $6 \mathrm{df}$ ], which was followed by a nonlinear registration to MNI152-2 $\mathrm{mm}$ space using FNIRT. Next, aCompCor (Napadow et al., 2010; Chai et al., 2012) was used to removed scanner-related and physiological noise from the fMRI using FSL, Matlab (MathWorks), and fMRISTAT (Worsley et al., 2002). Using FSL's FAST, we first segmented the T1-weighted anatomical images into partial volume gray matter (GM), white matter (WM), and CSF maps. The WM and CSF partial volume maps, which were derived 
from the subject's anatomical space, were linearly registered to their fMRI space. To prevent the possibility of removing any GM signal, the WM and CSF maps were thresholded to the top 198 and $20 \mathrm{~cm}^{3}$ probability of WM and CSF voxels, respectively, leaving the voxels with the highest probability of correctly being mapped as WM and CSF. This approach allowed us to exclude voxels that may have contained more than one tissue type (Chai et al., 2012). These thresholded maps were then multiplied by the resting-state fMRI data, and the top five WM and CSF components derived from a whole-brain principal component analysis were regressed out. The six motion parameters derived from MCFLIRT were also regressed out of the data, which was followed by spatial smoothing ( $6 \mathrm{~mm}$ full-width at half-maximum kernel). AFNI's $3 \mathrm{dBandpass}$ function was used to temporally filter the fMRI data into respective slow-wave frequency bands (slow-5, 0.01-0.027 Hz; slow-4, 0.027-0.073 Hz; slow-3, 0.073-0.198 Hz; Buzsaki and Draguhn, 2004; Di Martino et al., 2008; Zuo et al., 2010).

Definition of resting-state network/pathways of interest. Using the Harvard Oxford Cortical and Subcortical Atlases (Frazier et al., 2005; Desikan et al., 2006; Makris et al., 2006; Goldstein et al., 2007) and as per described previously (Rogachov et al., 2016), a mask of core regions of the ascending nociceptive system was created in the standard MNI152-2 $\mathrm{mm}$ space. This mask included the bilateral thalamus, bilateral primary and secondary somatosensory cortices $(\mathrm{S} 1, \mathrm{~S} 2)$, bilateral insula, and anterior and mid-cingulate cortices. We also included masks of the default mode network [DMN; medial prefrontal cortex, posterior cingulate/ precuneus $(\mathrm{PCC} / \mathrm{PCu})$, lateral temporal cortex, and lateral parietal cortex] and the salience network [SN; mid-cingulate cortex (MCC), dorsolateral prefrontal cortex (dlPFC), and temporoparietal junction; Hemington et al., 2016] because they are important in shaping an individual's pain experience and are key networks of the dynamic pain connectome (Kucyi and Davis, 2015).

Calculation of regional BOLD signal variability. BOLD signal variability/ LFOs was calculated on a voxel-wise basis using tools from FSL (Jenkinson et al., 2012). Specifically, using fslmaths, the SD of each voxel's time course was calculated for each subject. Next, since the global mean BOLD signal variability did not reveal significant group differences between AS patients and HCs $(p=0.34)$, each subject's BOLD signal variability map was standardized ( $z$-scored) on an individual basis (Martino et al., 2016). This was done to mitigate potential extraneous sources of variance in the BOLD signal between subjects (i.e., differences in magnetic gain between scans). The standardization process involved subtracting from each voxel the subject's mean BOLD signal variability across their gray matter and dividing this by the $\mathrm{SD}$ of the BOLD signal variability across the gray matter. Thus, a BOLD signal variability $z$-score value of 0 represents oscillations that are equal to the individual's average across the entire whole brain (gray matter). Positive values represent BOLD signal variability that is greater than the whole-brain (gray matter) average, and negative values represent those that are lower than the whole-brain (gray matter) average. The computed SD is related to the amplitude of the resting-state fluctuations in the time domain and reflects the strength or intensity of the low-frequency fluctuation (Zuo et al., 2010).

Group voxel-wise analysis. For each frequency band of interest, comparisons between the HC and AS groups' subject-level amplitude maps were performed using a two-sample unpaired $t$ test using Randomise, FSL's tool for nonparametric permutation inferencing (Winkler et al., 2014). We combined our masks/networks of interest (ascending nociceptive system, DMN, and SN) and ran voxel-wise permutation tests (positive and negative contrasts with 5000 permutations) with threshold-free cluster enhancement (TFCE). The presented group-level results are thresholded at $p<0.05$ [family-wise error (FWE) corrected for multiple comparisons]. Although there were no group differences in age ( $p=$ $0.194)$ and residual head motion $(p=0.533)$, previous studies have demonstrated that both factors can impact amplitude recordings (Garrett et al., 2010). Thus, to limit any possible confounding effects of age or head motion, both age and mean relative head displacement for each subject were included as variables of no interest in the design matrix.

Cross-validation regression: BOLD signal variability/LFOs and pain ratings. Using PRoNTo (Pattern Recognition for Neuroimaging Toolbox; Schrouff et al., 2013), we applied a machine learning approach to examine multivariate brain patterns that are related to patients' pain (Rogachov et al., 2015). To do this, voxel-wise BOLD signal variability was regressed against patients' pain ratings using cross-validation (10fold) kernel ridge regression. Of note, only the voxels that were statistically significant from the group-level BOLD signal variability analysis, between AS patients and HCs, were included in this analysis. Two separate models were created: one for patients' state pain intensity (current pain collected right before the MRI scan; $n=50$ ) and one for their trait-like pain intensity (average pain over the week; $n=56$ ). Follow-up permutation testing (5000 iterations) was conducted to determine the statistical significance of the correlation between actual and modelestimated pain ratings, as well as the mean squared error (MSE), the magnitude of error in the model.

Sex-related analyses. To examine whether the spatial distribution of the frequency-specific abnormalities we found in the main analysis were driven/explained by sex, we performed, within each frequency band of interest, a two-sample unpaired $t$ test on the subject-level amplitude maps for men [AS men $(n=19)$ vs HC men $(n=19)$ ] and women [AS women $(n=19)$ vs HC women $(n=19)]$ separately. The frequencyspecific group contrasts were masked and run through a voxel-wise permutation test (positive and negative contrasts with 5000 permutations) using TFCE. All reported results are thresholded at $p<0.05$ (FWE corrected).

Moreover, we examined sex differences within each condition (AS men vs AS women; HC men vs HC women) separately. The same networks (ascending nociceptive system, DMN, and SN) were amalgamated, masked, and run through a voxel-wise permutation test (positive and negative contrast with 5000 permutations) using TFCE. All reported results are thresholded at $p<0.05$ (FWE corrected).

\section{Results \\ BOLD signal variability is increased in AS chronic pain patients}

We found that compared with the HC group, AS patients had abnormally greater LFOs within regions of the dynamic pain connectome. Specifically, AS patients had increased LFOs within left ventrolateral thalamus and S1 of the ascending pain pathway in the slow-5 frequency band (Fig. 1). Within the slow-4 frequency band, AS patients exhibited increased LFOs in S1 of the ascending pain pathway and in the PCC and MCC of the DMN and SN, respectively (Fig. 1). Finally, patients had increased LFOs in S1 and $\mathrm{PCC} / \mathrm{PCu}$ compared with HCs in the slow-4 frequency band (Fig. 1). Within brain regions that showed statistically significant group differences, HCs had negative $z$-score values in slow- 5 and positive $z$-score values in slow-3 and slow-4. On the other hand, AS patients had positive $z$-score values in all frequency bands examined (Fig. 1, Table 1).

\section{BOLD signal variability impairments reflect trait-like pain ratings}

Of the 65 AS patients who provided an average pain intensity rating, 56 reported experiencing some pain in the week before testing (average week pain,1-10 of 10), while 9 of the patients (all of whom were undergoing treatment with biologic agents) reported being completely pain free. Only patients with pain were included in our analysis. The mean $( \pm S D)$ trait pain intensity rating over the last week of the AS patients was $4.03 \pm 2.18$. Using cross-validated multivariate regression, we found that a model based on LFOs could generalize to make inferences about unseen patients' average pain intensity ratings [trait pain; $n=56 ; r=$ $0.43, p=0.005$; MSE, $4.94(p=0.0088)$; Fig. 2]. The brain regions with the greatest predictive weights/magnitude to make inferences on unseen patients' for average pain ratings were the $\mathrm{PCC} / \mathrm{PCu}$ and right S1 (Fig. 2C). 

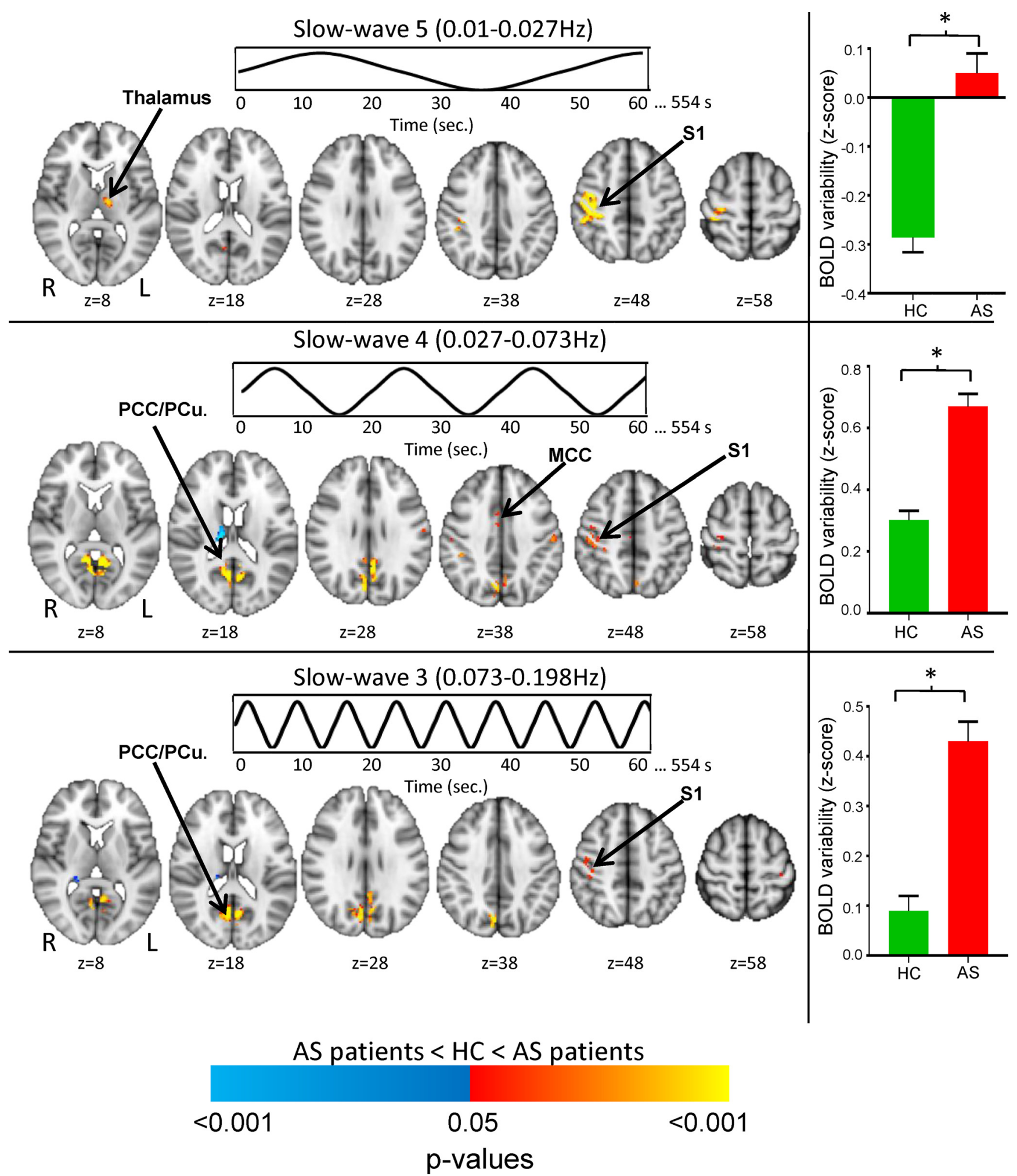

Figure 1. Frequency-specific changes in BOLD signal variability/LFOs. The contrastmaps represent the differences in BOLD signal variability/LFOs between $A S$ patients $(n=56)$ and HCs $(n=62)$ within three frequency bands: slow- $5(0.01-0.027 \mathrm{~Hz})$, slow- $4(0.027-0.073 \mathrm{~Hz})$, and slow-3 $(0.073-0.198 \mathrm{~Hz})$. Since there was no significant group differences in global BOLD variability $(p=0.34)$, subject-level standardization (z-scored) was performed. The corresponding raw Z-scored BOLD signal variability values averaged across the whole significant cluster are represented in the bar graphs. All statistical images display significant clusters ( $p<0.05$, FWE corrected). Warm colors represent brain areas in which AS patients had greater BOLD signal variability/LF0s than HCs, and cool colors represent brain areas in which AS patients had lower BOLD signal variability/LFOs than HCS. All axial slices are represented in radiological orientation. S1, Primary somatosensory cortex, MCC, Mid-cingulate cortex, and PCC/PCU - Posterior cingulate cortex/precuneus; L, left; R, right. ${ }^{*} p<0.05$, FWE-corrected.

Sex-related differences in BOLD signal variability

To investigate whether the abnormalities reported in our main result were driven by sex, a follow-up analysis was performed in men [AS $(n=19)$ vs HC $(n=19)]$ and in women [AS $(n=19)$ vs
HC $(n=19)]$, separately. Within the slowest frequency band (slow-5), the majority of the results (e.g., thalamus and right S1) are driven by women. Likewise, our main findings in slow- 3 with the $\mathrm{PCC} / \mathrm{PCu}$ are also primarily explained by women. On the 
Table 1. Peak MNI coordinates from brain regions that had significantly increased and decreased BOLD signal variability in AS patients $(n=56)$ compared with HCs $(n=62)$

\begin{tabular}{|c|c|c|c|c|c|c|}
\hline \multirow[b]{2}{*}{ Frequency band } & \multirow[b]{2}{*}{ Contrast } & \multirow{2}{*}{$\begin{array}{l}\text { Number of } \\
\text { voxels }\end{array}$} & \multicolumn{3}{|c|}{ MNI coordinates (mm) } & \multirow[b]{2}{*}{ Brain region } \\
\hline & & & $x$ & $y$ & $z$ & \\
\hline \multirow[t]{3}{*}{ Slow-5 $(0.01-0.027 \mathrm{~Hz})$} & $A S>H C$ & 441 & 42 & -28 & 42 & Right primary somatosensory cortex \\
\hline & & 67 & -10 & -14 & 8 & Left thalamus \\
\hline & & 4 & 6 & -60 & 16 & Posterior cingulate cortex \\
\hline \multirow[t]{7}{*}{ Slow-4 (0.027-0.073 Hz) } & $A S>H C$ & 1056 & 8 & -52 & 10 & Precuneus \\
\hline & & 291 & 46 & -36 & 42 & Right supramarginal gyrus \\
\hline & & 57 & -56 & -16 & 36 & Left primary somatosensory cortex \\
\hline & & 31 & 6 & -2 & 40 & Mid-cingulate cortex \\
\hline & & 10 & 62 & -6 & 24 & Right primary somatosensory cortex \\
\hline & $\mathrm{AS}<\mathrm{HC}$ & 99 & 16 & -26 & 16 & Right thalamus \\
\hline & & 13 & -14 & -28 & 16 & Left thalamus \\
\hline \multirow[t]{4}{*}{ Slow-3 $(0.073-0.198 \mathrm{~Hz})$} & $A S>H C$ & 598 & 6 & -70 & 34 & Precuneus \\
\hline & & 81 & 40 & -22 & 50 & Right primary somatosensory cortex \\
\hline & & 19 & 42 & -28 & 46 & Right primary somatosensory cortex \\
\hline & $A S<H C$ & 23 & 18 & -24 & 14 & Right thalamus \\
\hline
\end{tabular}

All statistical images were thresholded at $p<0.05$ (FWE corrected for multiple comparison) using TFCE.

A

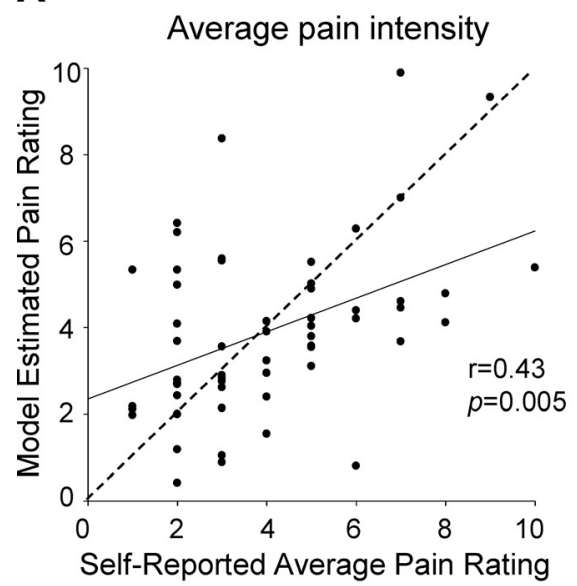

B

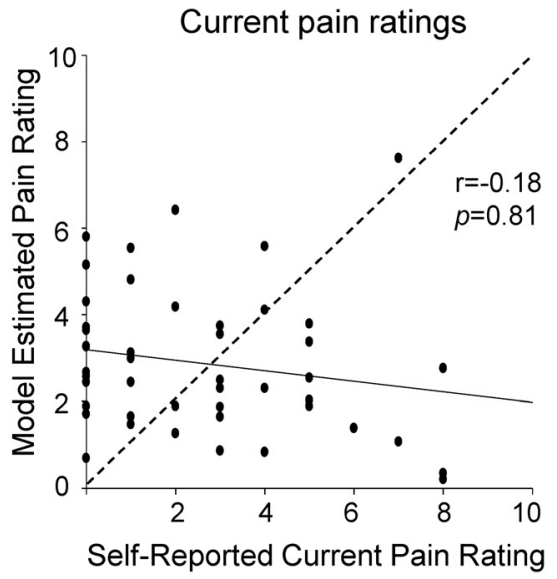

C Brain weight maps used for average pain inferences in unseen patients
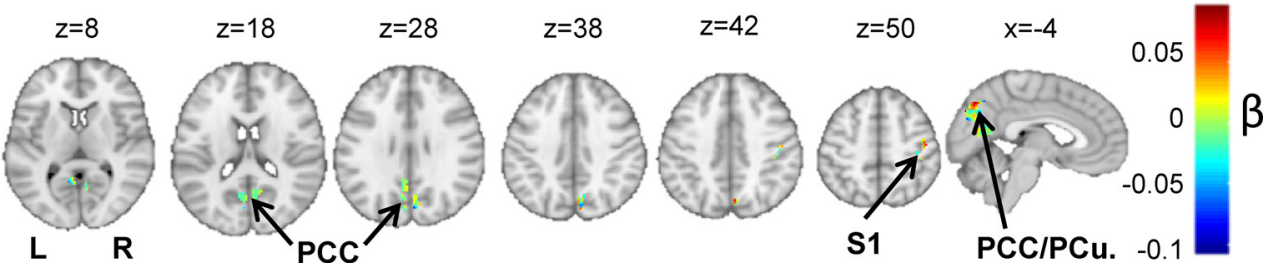

Figure 2. Group differences in BOLD signal variability/LFOs within slow-3 are generalizable for average pain intensity ratings. $A$, Positive, significant correlation between self-reported average pain intensity ratings and model-estimated average pain intensity ratings using BOLD signal variability/LF0s in areas (i.e., PCC and S1) that showed group differences in slow-3 between AS patients and HCs $(r=0.43, p=0.005, n=56) . \boldsymbol{B}$, No relationship between self-reported state pain ratings and model-estimated state pain ratings in areas that had group differences in slow-3 between AS patients and $\mathrm{HCS}(r=-0.18, p=0.81, n=50)$. C, The distribution of brain regions and their associated weights that were used in the machine learning model to make inferences about average pain intensity ratings. PCC, Posterior cingulate cortex; S1, Primary somatosensory cortex; L, Left; R, right.

other hand, slow- 4 results show to have been driven by both sexes fairly equally, with men accounting for a marginally larger proportion in the PCC/PCu. This suggests that the majority of our findings with our main analysis, particularly with slow-3 and slow-5, are explained by women subjects (Table 2, Fig. 3 ).

Furthermore, we explored sex differences within the subjects used in this study. Among the AS patients (19 men, 19 women), although both positive, men exhibited lower slow-5 BOLD signal variability/LFOs in the PCC compared with women (average \pm SEM: men, $0.35 \pm 0.08$; women, $0.90 \pm 0.07 ; p<0.05$ ). However, in the higher-frequency bands, men had higher, positive BOLD signal variability/LFOs than the women, who had negative BOLD signal variability/LFOs in dIPFC (slow-4; men, $0.42 \pm 0.19$; women, $-0.67 \pm 0.11$ ) and right primary S1 (slow-3; men, $0.09 \pm 0.11$; women, $-0.71 \pm 0.07 ; p<0.05$; Fig. 4 , Table 3 ). None of these effects were seen in HCs. Finally, there were no sex differences in trait pain $(z=-0.157, p=0.8858)$.

\section{State versus trait pain characteristics}

The temporal dimension of chronic pain is often overlooked, yet patients commonly report that their symptoms can fluctuate from moment to moment and even on the order of hours and days. Brain imaging studies of chronic pain typically examine brain function related to the amount of pain that a patient re- 
Table 2. Peak MNI coordinates from brain regions that show sex-related effects from the main analysis

\begin{tabular}{|c|c|c|c|c|c|c|}
\hline \multirow[b]{2}{*}{ Frequency band } & \multirow[b]{2}{*}{ Contrast } & \multirow{2}{*}{$\begin{array}{l}\text { Number of } \\
\text { voxels }\end{array}$} & \multicolumn{3}{|c|}{ MNI coordinates (mm) } & \multirow[b]{2}{*}{ Brain region } \\
\hline & & & $x$ & $y$ & $z$ & \\
\hline Slow-5 $(0.01-0.027 \mathrm{~Hz})$ & Men < women (AS patients) & 36 & -2 & -44 & 26 & Posterior cingulate cortex \\
\hline Slow-4 $(0.027-0.073 \mathrm{~Hz})$ & Men > women (AS patients) & 8 & -32 & 50 & 28 & Left dorsolateral prefrontal cortex \\
\hline Slow-3 $(0.073-0.198 \mathrm{~Hz}$ & Men > women (AS patients) & 9 & 48 & -28 & 62 & Right primary somatosensory cortex \\
\hline
\end{tabular}

The AS patients and HCs both consisted of 19 men and 19 women. All statistical images were thresholded at $p<0.05$ (FWE corrected for multiple comparison) using cluster-free threshold enhancement.

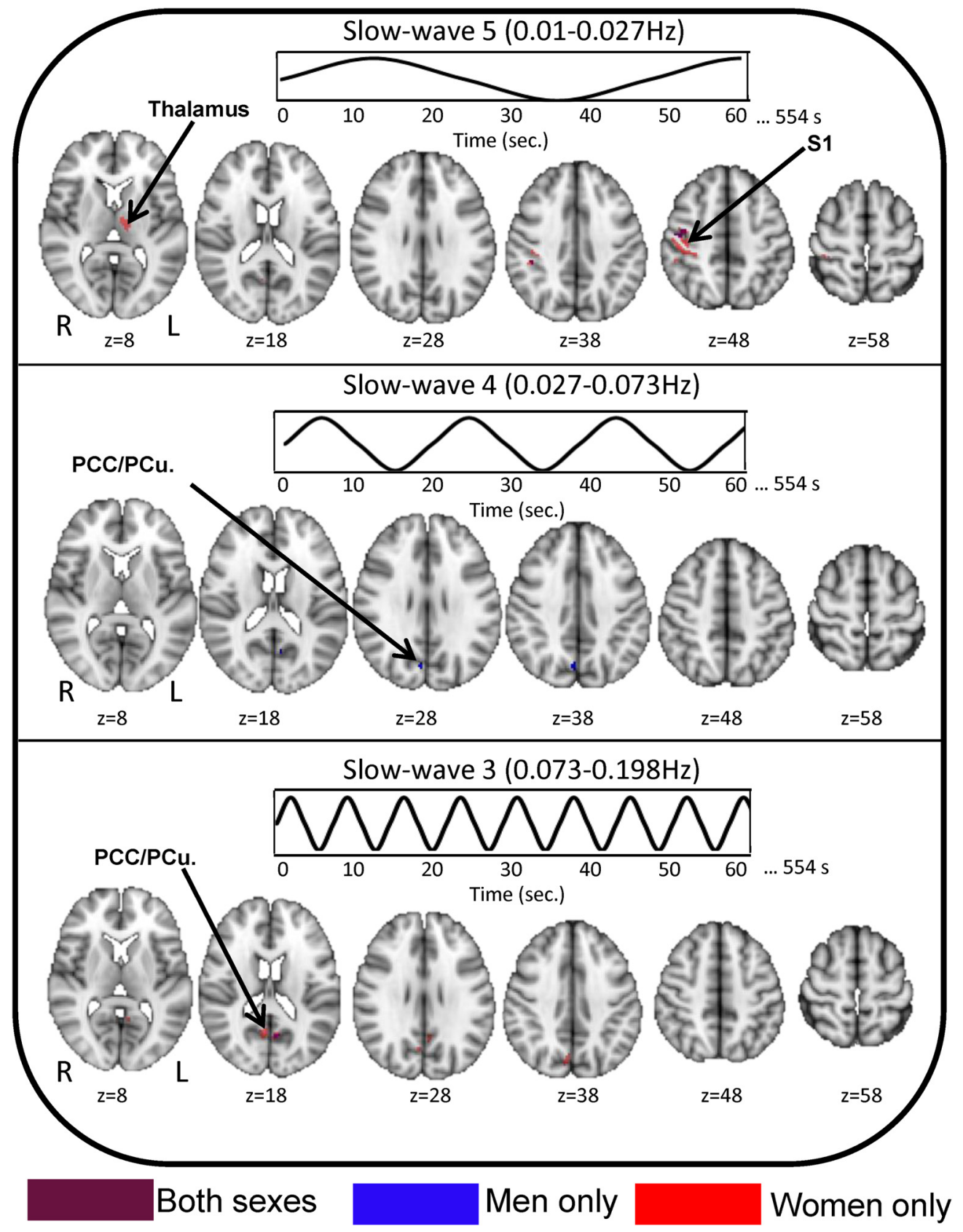

Figure 3. Frequency-specific differences in BOLD signal variability/LFOs between AS patients and HCs is primarily driven by female subjects. The contrast map indicates the extent to which the differences in BOLD signal variability/LFOs between all AS patients versus all HCs is explained by male subjects (male AS patients vs male HCs; blue clusters), by female subjects (AS patients vs female $\mathrm{HCS}$; red clusters), or by both sexes (purple clusters). All statistical images display significant clusters ( $p<0.05$, FWE corrected). All axial slices are represented in radiological orientation. S1, primary somatosensory cortex; PCC/PCu, Posterior cingulate cortex/precuneus.

ports. However, most studies do not distinguish pain at the time of the study (i.e., state pain) from average pain experienced over time (i.e., trait pain). Both state and trait pain reflect important but different aspects of chronic pain conditions. One of the sec- ondary aims of this study was to raise the issue that functional brain abnormalities commonly reported in chronic pain patients may not explain both state- and trait-like pain characteristics. Our results show that, even though state- and trait-like pain char- 


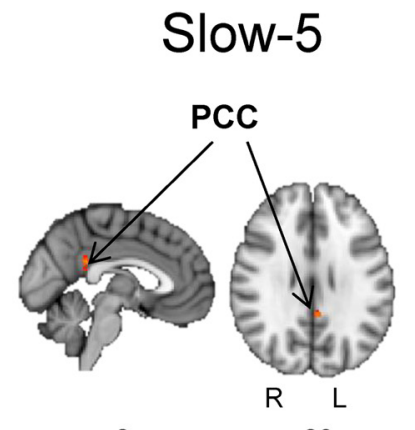

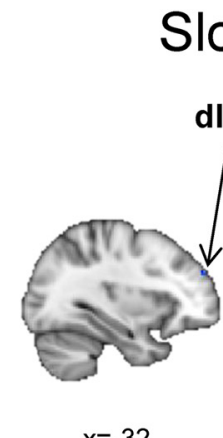

Slow-4

dIPFC

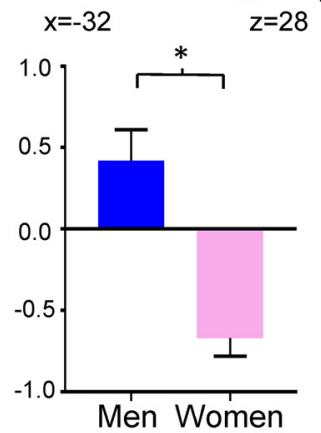

\section{Slow-3}
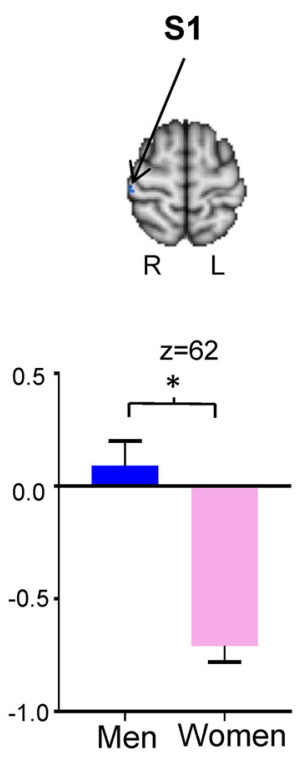

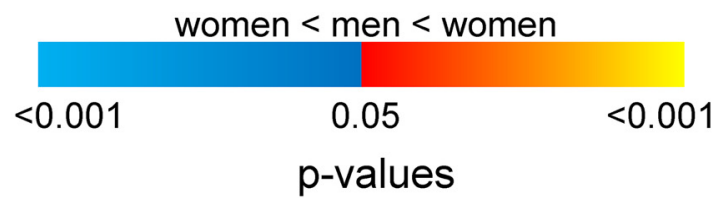

Figure 4. Frequency-specific sex differences in BOLD signal variability/LFOs in healthy controls and AS patients. The histograms (blue, men; pink, women) represent the standardized BOLD signal variability values ( $z$-scores) extracted from the significant clusters derived from the contrast maps between men and women. The significant clusters are depicted in the brain maps shown to the right of each graph. All significant clusters are thresholded at $p<0.05$ (FWE corrected). Warm colors represent brain areas in which women had greater BOLD signal variability/LFOs compared with men, and cool colors represent brain areas in which women had lower BOLD signal variability/LFOs compared with men. S1, Primary somatosensory cortex; dIPFC, Dorsolateral prefrontal cortex; PCC, Posterior cingulate cortex; ${ }^{*} p<0.05$, FWE-corrected.

Table 3. Peak MNI coordinates from brain regions that showed sex-differences in BOLD signal variability/LFOs

\begin{tabular}{|c|c|c|c|c|c|c|}
\hline \multirow[b]{2}{*}{ Frequency Band } & \multirow[b]{2}{*}{ Contrast } & \multirow{2}{*}{$\begin{array}{l}\text { Number of } \\
\text { voxels }\end{array}$} & \multicolumn{3}{|c|}{ MNI coordinates $(\mathrm{mm})$} & \multirow[b]{2}{*}{ Brain region } \\
\hline & & & $x$ & $y$ & $z$ & \\
\hline \multirow[t]{5}{*}{ Slow-5 $(0.01-0.027 \mathrm{~Hz})$} & $\mathrm{AS}$ men $>\mathrm{HC}$ men & 49 & 46 & -16 & 50 & Right primary somatosensory cortex \\
\hline & & 14 & 42 & -36 & 40 & Right supramarginal gyrus \\
\hline & & 10 & 38 & -20 & 54 & Right primary somatosensory cortex \\
\hline & AS women $>\mathrm{HC}$ women & 289 & 46 & -38 & 40 & Right primary somatosensory cortex \\
\hline & & 23 & -6 & -10 & 6 & Left thalamus \\
\hline Slow-4 (0.027-0.073 Hz) & $\mathrm{AS}$ men $>\mathrm{HC}$ men & 57 & 6 & -72 & 34 & Precuneus \\
\hline \multirow[t]{3}{*}{ Slow-3 $(0.073-0.198 \mathrm{~Hz})$} & AS men $>\mathrm{HC}$ men & 21 & -6 & -62 & 18 & Posterior cingulate cortex \\
\hline & AS women $>\mathrm{HC}$ women & 154 & 8 & -60 & 18 & Posterior cingulate cortex \\
\hline & & 90 & -6 & -60 & 16 & Posterior cingulate cortex \\
\hline
\end{tabular}

The AS patients consisted of 19 men and 19 women. All statistical images were thresholded at $p<0.05$ (FWE corrected for multiple comparison) using cluster-free threshold enhancement.

acteristics are highly correlated (rho $=0.732, p<0.001$ ), functional brain impairments, as revealed through LFO, cannot explain patients' current (state-like) pain intensity ratings (Fig. $2 B$ ).

\section{Additional analysis: impact of head motion, psychiatric metrics, and treatment effects}

One important consideration of this study is the potential of physiological rhythms and head motion artifacts being aliased into the BOLD signal, particularly at higher frequencies (Cordes et al., 2001). We performed additional analyses to examine whether any of our findings were impacted by technical factors (i.e., head motion) or by psychiatric and treatment effects.

There was no significant differences in mean relative framewise displacement between AS patients and HCs (HCs, $0.09 \pm$
$0.06 \mathrm{~mm}$; AS patients, $0.07 \pm 0.04 \mathrm{~mm}$; independent-samples Mann-Whitney $U$ test, $z=-0.128, p=0.898$ ). Also, including mean relative head motion as a covariate of no interest into the multivariate regression model had a marginal effect on the ability of slow-3 LFOs to make generalizable inferences about patients' trait pain $(r=0.43, p=0.0082$; MSE, $4.94 ; p=0.0126)$.

As expected, there were statistically significant differences between the patients and HCs in psychiatric metrics, specifically depression (BDI: $z=-3.98, p<0.05)$, state anxiety $(z=-4.25$, $p<0.05)$, and trait anxiety $(z=-3.73, p<0.05)$, although none of the patients had clinical levels indicative of a psychiatric disorders. However, we did not find any significant relationship between BOLD signal variability/LFO group contrast results in any of the frequency bands and these psychiatric measures (slow-5: 
BDI, $n=56$, Spearman's rho $=0.02, p=0.886$; state anxiety, $n=$ 56 , rho $=-0.216, p=0.11$; trait anxiety, $n=56$, rho $=-0.048$, $p=0.726$; slow-4: BDI, $n=56$, rho $=-0.069, p=0.613$; state anxiety, $n=56$, rho $=0.005, p=0.973$; trait anxiety, $n=56$, rho $=-0.048, p=0.726$; slow- 3 : BDI, $n=56$, rho $=-0.043$, $p=0.753$; state anxiety, $n=56$, rho $=0.043, p=0.755$; trait anxiety, $n=56$, rho $=-0.024, p=0.858)$. Therefore, it is unlikely that the relationship between BOLD signal variability and patients' average pain are driven by anxiety or depression.

Finally, since our cohort contained patients both treated $(n=$ $31)$ and nontreated $(n=25)$ with biologics, we performed a follow-up analysis to ensure that our results were not driven by treatment. Within the clusters that showed significant group differences between AS patients and HCs, treated and nontreated patients did not show significant differences in BOLD signal variabililty/LFOs within any of the tested frequency bands [mean \pm SEM BOLD signal variability $(z-$ scores); slow-5: treated, $-0.122 \pm 0.06$; nontreated, $-0.04 \pm$ $0.07 ; z=-0.700, p=0.721$; slow 4: treated, $0.65 \pm 0.06$; nontreated, $0.69 \pm 0.06 ; z=-0.783, p=0.883$; slow 3: treated, $0.41 \pm 0.06$; nontreated, $0.46 \pm 0.07 ; z=-0.882$, $p=0.383]$.

\section{Discussion}

This is the first study to use a multivariate, machine learning approach to examine LFOs in the dynamic pain connectome of patients with chronic pain. Our main finding is that chronic pain patients with AS exhibit higher-frequency-specific aberrations in BOLD signal variability/LFOs compared with HCs. Within slower frequencies (slow-5), patients had abnormal LFOs in the ascending pain pathway, whereas at higher frequencies (slow-4 and slow-3) these abnormalities were also found in the DMN and $\mathrm{SN}$. Furthermore, our machine learning algorithm revealed that the pattern of slow-3 LFO abnormalities can be used to make generalizable inferences about average pain intensity ratings in previously unseen patients.

Our finding that patients with chronic pain have higher brain signal variability in the dynamic pain connectome (i.e., within the ascending pain pathway, $\mathrm{DMN}$, and $\mathrm{SN}$ ) are in line with previous studies of LFOs (Baliki et al., 2011, 2014; Alshelh et al., 2016; Hodkinson et al., 2016). These findings could arise through several mechanisms. First, increased resting-state LFOs within the ascending pain pathway could result from the heightened level of sensory traffic that manifests as part of the ongoing, fluctuating chronic pain intensity. This is supported by other studies in neuropathic pain patients that found increased LFOs within the thalamus and S1 within a similar frequency range $(0.04 \mathrm{~Hz}$; slow5-slow-4 border; Alshelh et al., 2016), suggesting that this effect is generalizable to many chronic pain conditions. Pain-related rumination and negatively valenced internal thought are other factors that could contribute to increased BOLD signal variability/ LFOs. For example, activity in the DMN has previously been attributed to episodes of internally directed and self-referential thought (Fox and Raichle, 2007). Disruption of the DMN has been shown across multiple chronic pain populations (Baliki et al., 2008; Napadow et al., 2010), as well as abnormal increases in LFOs in the DMN of chronic low back pain patients (Baliki et al., 2008). Our patients also exhibited heightened LFOs in the MCC, an area associated with pain affect (Vogt, 2005). The MCC receives projections from the amygdala (Phan et al., 2002), a region implicated in fear (Whalen et al., 1998) and nociception (Bernard et al., 1992). Given the convergence of fear- and pain-related input into the MCC, its elevated engagement in chronic pain is thought to relate to avoidance behavior often reported in these patients (Vogt, 2005). Together, a heightened level of restingstate activity in the DMN and MCC could reflect the affectivemotivational aspect of AS chronic pain.

The neurobiological mechanisms underlying LFOs in the brain are unknown but could involve neuronal and nonneuronal activity (Y. F. Wang et al., 2014; Alshelh et al., 2016). Preclinical studies have shown that low-frequency brain oscillations, within the $0.1-0.5 \mathrm{~Hz}$ frequency range, are generated primarily through perpetual excitation within the cerebral cortex (Sanchez-Vives and McCormick, 2000) and maintained by rhythmic depolarization-hyperpolarization sequence via regulated excitatory and inhibitory postsynaptic potentials (Steriade et al., 1993). The continuous yet fluctuating influx of unpleasant, sensory input experienced by chronic pain patients may be related to the elevated LFOs responsible for the sensory discriminatory and affective-motivation aspects of pain. Moreover, others have speculated that non-neuronal cells (astrocytes) may contribute to LFO regulation and maintenance (Bernard et al., 1992; Alshelh et al., 2016).

Our study also examined the relationship between LFOs and average (trait) pain intensity but would also like to put forth the importance of studying pain characteristics on different time scales. Few studies have examined brain mechanisms underlying shorter-lasting (state) and longer-lasting (trait) chronic pain, and those that have explored this topic have reported conflicting results. For example, Baliki et al. (2011) showed that when spontaneous pain ratings are collected simultaneously with brain imaging data from chronic low back pain patients, the fluctuating patterns of the pain ratings followed a similar time course as the hemodynamic response from which the LFOs are derived, an effect that was only present in the upper slow-3 range (0.12-0.2 $\mathrm{Hz}$ ). However, more recently the same group also showed that increased LFO amplitudes (upper half of slow-3, 0.12-0.2 Hz) in the DMN were correlated with the duration of pain in low back pain and osteoarthritis patients, proposing a relationship between LFOs and trait-like features (Baliki et al., 2014). In line with these studies, we found a relationship between higher-frequency LFOs (slow-3) and average pain intensity. However, unlike the aforementioned studies, we used a robust, multivariate machine learning technique that highlights the generalizability between slow-3 LFO abnormalities and average pain intensity ratings. Similar to Baliki et al. (2014), our brain weight maps also suggested that slow-3 LFO abnormalities within DMN (PCC) holds the greatest prediction power for trait pain rating in unseen patients (Fig. 2C).

The mechanisms underlying state- and trait-like chronic pain features may not necessarily be mutually exclusive but, nevertheless, pose a crucial consideration when investigating neural correlates of chronic pain characteristics. The current findings complement our recent work showing a unique pattern of brain connectivity that can be used to differentiate state and trait pain (Cheng et al., 2018). Trait pain ratings reflect intensity levels that act as an anchor around which state pain levels fluctuate throughout the course of the day. Our findings suggest that aberrations in brain mechanisms that are present in chronic pain patients are reflective of the persistent, longer-lasting intensity of pain and not shorter, state-like representations. The brain plasticity that is responsible for the manifestation of impaired brain mechanisms that develop as a result of chronic pain symptoms presumably occurs over the course of multiple days/weeks and, therefore, is unable to track a single state-like rating that is bound to fluctuate throughout the course of the day. Thus, it is important to con- 
sider not only pain intensity levels during testing but also across ratings over longer time scales, which may actually be more informative when investigating brain mechanisms. Of note, there are a number of different approaches to assess trait-like pain characteristics. In this study, patients provided one rating to represent their average pain rating in the week before testing on test date. An alternative approach could have been to have patients use a pain diary to document their pain levels multiple times per day in the week before our testing. Both approaches have advantages and disadvantages, but our straightforward approach was made primarily for practical and compliance issues and is one commonly used by clinicians to obtain a general sense of a patient's pain.

Many patients with chronic pain can have comorbidity with various psychiatric disorders. Although we screened patients and excluded those with a confirmed psychiatric diagnosis, some of our patients had increased levels of anxiety (state and trait) and depression scores compared with the HCs. Therefore, we also investigated whether our main study findings can be explained by these psychiatric effects. Overall, all three traits demonstrated a low correlation with BOLD signal variability group differences in each of the frequency bands, thus limiting the possibility that these effects are driven by any psychiatric involvement and emphasizing their specificity to pain severity.

A secondary aim of this study was to examine sex-related effects. First, we found that the majority of the results obtained from our main analysis, particularly with slow- 3 and slow- 5 , are primarily driven by women subjects. Compared with male patients, women patients show greater functional impairments in pain-associated brain regions, including the ascending pain pathway, compared with their respective controls. Since no sexdifferences in average pain ratings exist, this finding suggests that women may have greater engagement of the antinociceptive system to offset these differences. Although the current study did not specifically probe the antinociceptive system, our previous work has shown that, compared with men, women have stronger functional connectivity between the subgenual cingulate cortex and the periaqueductal gray, proposing a greater engagement of the descending modulation system in women (G. Wang et al., 2014). Second, unlike in HCs, our findings suggest frequency-specific sex differences in BOLD oscillations in AS patients, which are consistent with previous studies showing decreased amplitude of LFOs at higher frequencies in the sensorimotor cortex in women compared with men with chronic pain (Hong et al., 2013; Fig. 4). Whereas the neurophysiological significance behind these discrepancies is unclear, it may suggest sex-specific sensory discrimination/perception in chronic pain patients.

In conclusion, we report that patients with chronic pain exhibit frequency-specific abnormalities in LFOs within the dynamic pain connectome as revealed through BOLD signal variability/LFOs, an effect that was not impacted by technical (i.e., head motion) or psychiatric (i.e., depression, anxiety) factors. These abnormalities were localized to the ascending pain pathway at slow oscillation frequencies (slow-5) but also engaged the DMN and SN at higher frequencies (slow- 4 and slow-3). Aberrations in BOLD signal variability/LFOs in slow- 3 could be used to make generalizable inference about patients' average pain intensity ratings. Thus, our findings indicate that measurable impairments in brain activity in chronic pain patients reflect persistent, longer-lasting levels of trait pain, as opposed to shorter-scale state-like pain.

\section{References}

Alshelh Z, Di Pietro F, Youssef AM, Reeves JM, Macey PM, Vickers ER, Peck CC, Murray GM, Henderson LA (2016) Chronic neuropathic pain: it's about the rhythm. J Neurosci 36:1008-1018. CrossRef Medline

Baliki MN, Geha PY, Apkarian AV, Chialvo DR (2008) Beyond feeling: chronic pain hurts the brain, disrupting the default-mode network dynamics. J Neurosci 28:1398-1403. CrossRef Medline

Baliki MN, Baria AT, Apkarian AV (2011) The cortical rhythms of chronic back pain. J Neurosci 31:13981-13990. CrossRef Medline

Baliki MN, Mansour AR, Baria AT, Apkarian AV (2014) Functional reorganization of the default mode network across chronic pain conditions. PLoS One 9:e106133. CrossRef Medline

Beck AT, Ward CH, Mendelson M, Mock J, Erbaugh J (1961) An inventory for measuring depression. Arch Gen Psychiatry 4:561-571. CrossRef Medline

Bernard JF, Huang GF, Besson JM (1992) Nucleus centralis of the amygdala and the globus pallidus ventralis: electrophysiological evidence for an involvement in pain processes. J Neurophysiol 68:551-569. CrossRef Medline

Bidad K, Gracey E, Hemington KS, Mapplebeck JCS, Davis KD, Inman RD (2017) Pain in ankylosing spondylitis: a neuro-immune collaboration. Nat Rev Rheumatol 13:410-420. CrossRef Medline

Buzsaki G, Draguhn A (2004) Neuronal oscillations in cortical networks. Science 304:1926-1929. CrossRef Medline

Chai XJ, CastañónAN, OngürD, Whitfield-Gabrieli S (2012) Anticorrelations in resting state networks without global signal regression. Neuroimage 59:1420-1428. CrossRef Medline

Cheng JC, Rogachov A, Hemington KS, Kucyi A, Bosma RL, Lindquist MA, Inman RD, Davis KD (2018) Multivariate machine learning distinguishes cross-network dynamic functional connectivity patterns in state and trait neuropathic pain. Pain. Advance online publication. Retrieved Apr 26, 2018. doi:10.1097/j.pain.0000000000001264.

Cordes D, Haughton VM, Arfanakis K, Carew JD, Turski PA, Moritz CH, Quigley MA, Meyerand ME (2001) Frequencies contributing to functional connectivity in the cerebral cortex in "resting-state" data. Am J Neuroradiol 22:1326-1333. Medline

Desikan RS, SégonneF, Fischl B, Quinn BT, Dickerson BC, Blacker D, Buckner RL, Dale AM, Maguire RP, Hyman BT, Albert MS, Killiany RJ (2006) An automated labeling system for subdividing the human cerebral cortex on MRI scans into gyral based regions of interest. Neuroimage 31:968980. CrossRef Medline

Di Martino A, Ghaffari M, Curchack J, Reiss P, Hyde C, Vannucci M, Petkova E, Klein DF, Castellanos FX (2008) Decomposing intra-subject variability in children with attention-deficit/hyperactivity disorder. Biol Psychiatry 64:607-614. CrossRef Medline

Fox MD, Raichle ME (2007) Spontaneous fluctuations in brain activity observed with functional magnetic resonance imaging. Nat Rev Neurosci 8:700-711. CrossRef Medline

Frazier JA, Chiu S, Breeze JL, Makris N, Lange N, Kennedy DN, Herbert MR, Bent EK, Koneru VK, Dieterich ME, Hodge SM, Rauch SL, Grant PE, Cohen BM, Seidman LJ, Caviness VS, Biederman J (2005) Structural brain magnetic resonance imaging of limbic and thalamic volumes in pediatric bipolar disorder. Am J Psychiatry 162:1256-1265. CrossRef Medline

Garrett DD, Kovacevic N, McIntosh AR, Grady CL (2010) Blood oxygen level-dependent signal variability is more than just noise. J Neurosci 30: 4914-4921. CrossRef Medline

Goldstein JM, Seidman LJ, Makris N, Ahern T, O'Brien LM, Caviness VS Jr, Kennedy DN, Faraone SV, Tsuang MT (2007) Hypothalamic abnormalities in schizophrenia: sex effects and genetic vulnerability. Biol Psychiatry 61:935-945. CrossRef Medline

Hemington KS, Wu Q, Kucyi A, Inman RD, Davis KD (2016) Abnormal cross-network functional connectivity in chronic pain and its association with clinical symptoms. Brain Struct Funct 221:4203-4219. CrossRef Medline

Hodkinson DJ, Wilcox SL, Veggeberg R, Noseda R, Burstein R, Borsook D, Becerra L (2016) Increased amplitude of thalamocortical low-frequency oscillations in patients with migraine. J Neurosci 36:8026-8036. CrossRef Medline

Hong JY, Kilpatrick LA, Labus J, Gupta A, Jiang Z, Ashe-McNalley C, Stains J, Heendeniya N, Ebrat B, Smith S, Tillisch K, Naliboff B, Mayer EA (2013) Patients with chronic visceral pain show sex-related alterations in intrin- 
sic oscillations of the resting brain. The Journal of neuroscience: the official journal of the Society for Neuroscience 33:11994-12002.

Jenkinson M, Beckmann CF, Behrens TE, Woolrich MW, Smith SM (2012) FSL. Neuroimage 62:782-790. CrossRef Medline

Kucyi A, Davis KD (2015) The dynamic pain connectome. Trends Neurosci 38:86-95. CrossRef Medline

Lutkenhoff ES, Rosenberg M, Chiang J, Zhang K, Pickard JD, Owen AM, Monti MM (2014) Optimized brain extraction for pathological brains (optiBET). PLoS One 9:e115551. CrossRef Medline

Makris N, Goldstein JM, Kennedy D, Hodge SM, Caviness VS, Faraone SV, Tsuang MT, Seidman LJ (2006) Decreased volume of left and total anterior insular lobule in schizophrenia. Schizophr Res 83:155-171. CrossRef Medline

Martino M, Magioncalda P, Huang Z, Conio B, Piaggio N, Duncan NW, Rocchi G, Escelsior A, Marozzi V, Wolff A, Inglese M, Amore M, Northoff G (2016) Contrasting variability patterns in the default mode and sensorimotor networks balance in bipolar depression and mania. Proc Natl Acad Sci U S A 113:4824-4829. CrossRef Medline

Napadow V, LaCount L, Park K, As-Sanie S, Clauw DJ, Harris RE (2010) Intrinsic brain connectivity in fibromyalgia is associated with chronic pain intensity. Arthritis Rheum 62:2545-2555. CrossRef Medline

Phan KL, Wager T, Taylor SF, Liberzon I (2002) Functional neuroanatomy of emotion: a meta-analysis of emotion activation studies in PET and fMRI. Neuroimage 16:331-348. CrossRef Medline

Rogachov A, Cheng JC, DeSouza DD (2015) Discriminating neural representations of physical and social pains: how multivariate statistics challenge the "shared representation" theory of pain. Journal of neurophysiology 114:2558-2560. CrossRef Medline

Rogachov A, Cheng JC, Erpelding N, Hemington KS, Crawley AP, Davis KD (2016) Regional brain signal variability: a novel indicator of pain sensitivity and coping. Pain 157:2483-2492. CrossRef Medline

Sanchez-Vives MV, McCormick DA (2000) Cellular and network mechanisms of rhythmic recurrent activity in neocortex. Nat Neurosci 3:10271034. CrossRef Medline

Schrouff J, Rosa MJ, Rondina JM, Marquand AF, Chu C, Ashburner J, Phillips C, Richiardi J, Mourão-MirandaJ (2013) PRoNTo: pattern recognition for neuroimaging toolbox. Neuroinformatics 11:319-337. CrossRef Medline

Sieper J, Braun J, Rudwaleit M, Boonen A, Zink A (2002) Ankylosing spondylitis: an overview. Ann Rheum Dis 61 [Suppl 3]:iii8-18.

Spielberger CD, Gorsuch RL, Lushene R, Vagg PR, Jacobs GA (1983) Manual for the State-Trait Anxiety Inventory. Palo Alto, CA: Consulting Psychologists.

Steriade M, NuñezA, Amzica F (1993) A novel slow ( $<1 \mathrm{~Hz}$ ) oscillation of neocortical neurons in vivo: depolarizing and hyperpolarizing components. J Neurosci 13:3252-3265. CrossRef Medline

van der Linden S, Valkenburg HA, Cats A (1984) Evaluation of diagnostic criteria for ankylosing spondylitis. A proposal for modification of the New York criteria. Arthritis Rheum 27:361-368. CrossRef Medline

Vogt BA (2005) Pain and emotion interactions in subregions of the cingulate gyrus. Nat Rev Neurosci 6:533-544. CrossRef Medline

Wang G, Erpelding N, Davis KD (2014) Sex differences in connectivity of the subgenual anterior cingulate cortex. Pain 155:755-763. CrossRef Medline

Wang YF, Liu F, Long ZL, Duan XJ, Cui Q, Yan JH, Chen HF (2014) Steadystate BOLD response modulates low frequency neural oscillations. Sci Rep 4:7376. Medline

Whalen PJ, Rauch SL, Etcoff NL, McInerney SC, Lee MB, Jenike MA (1998) Masked presentations of emotional facial expressions modulate amygdala activity without explicit knowledge. J Neurosci 18:411-418. CrossRef Medline

Winkler AM, Ridgway GR, Webster MA, Smith SM, Nichols TE (2014) Permutation inference for the general linear model. Neuroimage 92:381-397. CrossRef Medline

Worsley KJ, Liao CH, Aston J, Petre V, Duncan GH, Morales F, Evans AC (2002) A general statistical analysis for fMRI data. Neuroimage 15:1-15. CrossRef Medline

Wu Q, Inman RD, Davis KD (2013) Neuropathic pain in ankylosing spondylitis: a psychophysics and brain imaging study. Arthritis Rheum 65: 1494-1503. CrossRef Medline

Zuo XN, Di Martino A, Kelly C, Shehzad ZE, Gee DG, Klein DF, Castellanos FX, Biswal BB, Milham MP (2010) The oscillating brain: complex and reliable. Neuroimage 49:1432-1445. CrossRef Medline 